\title{
Control of glycemia and other cardiovascular disease risk factors in older adults with type 2 diabetes mellitus: data from the adult diabetes control and management
}

\begin{abstract}
Aim: The aims of the present study were to assess the control of glycemia and other cardiovascular disease risk factors, and the association between age and these controls among older adults with type 2 diabetes in Malaysia.

Methods: A cross-sectional study was carried out using cases notified to the Adult Diabetes Control and Management database between 1 January and 31 December 2009. A total of 10363 people aged over 60 years with type 2 diabetes mellitus were included in the analyses. A standard online case report form was used to record demographic data, clinical factors (diabetes duration, comorbid condition and treatment modalities), cardiovascular disease risk factors, diabetes complications and laboratory assessments. The cardiovascular disease risk factors controls assessed included glycosylated hemoglobin (HbA1c) $<7.0 \%$, blood pressure, body mass index, waist circumference and lipid profiles.

Results: The proportion of older adults who achieved target HbA1c $(<7.0 \%)$ was $41.7 \%$. A greater proportion of older adults aged $\geq 80$ years significantly achieved the targets of $\mathrm{HbA} 1 \mathrm{c}$ $<7 \%$ ( $\mathrm{P}<0.001)$, waist circumference $(\mathrm{P}<0.001)$, low-density lipoprotein cholesterol $<2.6 \mathrm{mmol} / \mathrm{L}(\mathrm{P}=0.007)$ and triglycerides $<1.7 \mathrm{mmol} / \mathrm{L}(\mathrm{P}=0.001)$ when compared with the younger elderly groups. They were also associated with achieving target $\mathrm{HbA1c}<7.0 \%$ $(\mathrm{OR}=1.90,95 \% \mathrm{CI} 1.68-2.26)$ and triglycerides $<1.7 \mathrm{mmol} / \mathrm{L}(\mathrm{OR}=1.20,95 \% \mathrm{CI} 1.04-$ 1.46) than those aged 60-69 years.

Conclusion: The control of cardiovascular disease risk factors was suboptimal in older adults with type 2 diabetes. The oldest elderly were more likely to achieve target HbA1c $(<7.0 \%)$ and triglycerides $(<1.7 \mathrm{mmol} / \mathrm{L})$ than older adults aged $60-69$ years.
\end{abstract}

Keyword: Elderly; Glycemic control; Malaysia; Registry; Type 2 diabetes mellitus 